\title{
Philosophiques
}

\section{La participation problématique des mathématiques à la dialectique}

\section{André Lebel}

Volume 37, numéro 1, printemps 2010

Albert Lautman, philosophe des mathématiques

URI : https://id.erudit.org/iderudit/039717ar

DOI : https://doi.org/10.7202/039717ar

Aller au sommaire du numéro

Éditeur(s)

Société de philosophie du Québec

ISSN

0316-2923 (imprimé)

1492-1391 (numérique)

Découvrir la revue

Citer cet article

Lebel, A. (2010). La participation problématique des mathématiques à la dialectique. Philosophiques, 37(1), 163-167. https://doi.org/10.7202/039717ar
Résumé de l'article

On propose ici trois avenues de recherche susceptibles d'élucider le lien un peu mystérieux entre dialectique et mathématiques dans la philosophie d'Albert Lautman. d'utilisation que vous pouvez consulter en ligne.

https://apropos.erudit.org/fr/usagers/politique-dutilisation/ 


\title{
La participation problématique des mathématiques à la dialectique
}

\author{
ANDRÉ LEBEL \\ Cégep Champlain St. Lawrence, Québec \\ alebel@slc.qc.ca
}

\begin{abstract}
RÉSUMÉ. - On propose ici trois avenues de recherche susceptibles d'élucider le lien un peu mystérieux entre dialectique et mathématiques dans la philosophie d'Albert Lautman.
\end{abstract}

ABSTRACT. - This note proposes three lines of work that might help to shed some light on the problematic nexus between dialectical ideas and mathematical theories in Albert Lautman's thinking.

\section{Introduction}

Le problème fondamental auquel doit faire face toute interprétation de la pensée d'Albert Lautman consiste à éclaircir le rapport du mathématique, en tant qu'ensemble de faits, d'êtres et de théories, à une dialectique dominatrice, rapport que Lautman conçoit comme constitutif de la réalité mathématique comme telle. Car, malgré de multiples, savantes et fascinantes tentatives, il semble bien que Lautman n'ait pas vraiment réussi à en rendre compte d'une manière pleinement satisfaisante. Un certain flottement terminologique, une évolution par exemple dans l'emploi des termes "concret» et «abstrait» d'un texte à l'autre, vient ajouter aux considérables difficultés intrinsèques que soulève cette question. Le respect, voire l'admiration, que plusieurs générations de mathématiciens portèrent jadis et portent encore de nos jours à l'œuvre de Lautman ne saurait faire oublier l'expression fréquente d'un certain scepticisme quant aux parties proprement philosophiques de son travail. On le constatera par exemple en relisant le compte rendu de la séance de la Société française de philosophie tenue en 1939, remarquable de par la présence de nombreux mathématiciens de très grande valeur (Maurice Fréchet, Élie Cartan, Paul Lévy et le jeune Charles Ehresmann, un formidable quatuor d'esprits mathématiques). Ce flottement, ces hésitations sont, il va sans dire, à mettre au compte de la brièveté de la carrière du philosophe, et il faut absolument tirer de cette tragédie quasi galoisienne tout le bien qu'il est possible, car il est très vrai - comme Lautman le rappelle lui-même à propos des sciences - que « les théories essayées et à peine ébauchées sont aussi fructueuses que les résultats définitifs ${ }^{1}$ ».

Pour faire progresser la compréhension du rapport entre dialectique et mathématiques, je propose aux philosophes et mathématiciens que cette

1. Lautman, 2006, p. 46. À moins d'indication contraire, toutes les notes renvoient à Lautman, Albert, Les mathématiques, les idées et le réel physique, Paris, Vrin, 2006. 
œuvre intéresse trois pistes, trois voies de recherche qui, si elles sont toutes les trois esquissées ou même essayées par Lautman dans ses textes, mériteraient à mon avis de plus amples développements et surtout plus précis.

\section{La voie «heideggerienne»}

C'est la plus difficile à décrire et à pratiquer pour le mathématicien que je suis, et le concours des philosophes sera donc déterminant. Il s'agirait en gros de comparer le «rapport originaire» qui existe toujours entre la réalitéhumaine (traduction de Dasein par Henri Corbin en 1938) et un monde, avec la relation problématique qui existerait entre dialectique et mathématiques. C'est Lautman lui-même qui, en 1939, suggère cette analogie:

Heidegger décrit ainsi, comme nous venons de la voir, une genèse du concept ontique de Monde à partir de l'idée de la réalité humaine; son intérêt primordial se porte donc sur le problème du Moi, mais ce primat des préoccupations anthropologiques dans sa philosophie ne saurait empêcher sa conception de la genèse des notions relatives à l'Existant, au sein de l'analyse des Idées relatives à l'Être, d'avoir une portée très générale ${ }^{2}[\ldots]$ on peut, à propos des rapports de la Dialectique et des mathématiques, serrer de près le mécanisme de cette opération où l'analyse des Idées se prolonge en création effective, où le virtuel se transforme en réel ${ }^{3}$.

Ainsi, Heidegger a donné des descriptions qui, en dépit de leur caractère "anthropologique ", sont néanmoins fort précises. Il faut espérer qu'un retour sur ces descriptions heideggeriennes permettra de clarifier ce que Lautman entend par "analyse ontologique", et contribuera enfin à déterminer avec plus de sûreté si la transposition ou l'analogie que propose Lautman dans son essai de 1939 tient bien la route 4 . Une conclusion négative serait à cet égard tout aussi intéressante qu'une conclusion positive. Une conclusion positive serait un enrichissement pour les études lautmaniennes et peut-être, qui sait, pour les études heideggeriennes, s'il est vrai que les

2. Ibid., p. 240. Je souligne.

3. Ibid., p. 242.

4. Cette "analyse ontologique» n'est peut-être rien d'autre que l'« eidétique descriptive » invoquée par Lautman durant le débat de 1939. Comme l'a souligné récemment Charles Alunni, les interprètes n'accordent pas à cette question toute l'importance qu'elle mérite (Alunni, Charles, "Albert Lautman et le souci brisé du mouvement", Revue de Synthèse, $5^{\mathrm{e}}$ série, 2005, p. 283-301). Une lettre de Cavaillès à Lautman montre bien que celui-ci ne pouvait ignorer le risque de contre-sens dans sa lecture de Heidegger $(\mathrm{H}$. Sinaceur, «Lettres inédites de Jean Cavaillès à Albert Lautman", Revue d'histoire des sciences, XL (1) 1987, p. 123). Il me semble qu'avant de rejeter en bloc l'interprétation que Lautman donne de Heidegger il faudrait analyser sérieusement l'essai Vom Wesen des Grundes dans le contexte de la pensée du Heidegger de la fin des années vingt. À cette époque, loin d'afficher l'anti-platonisme qui fut plus tard le sien, Heidegger "n'hésite [...] aucunement à se solidariser avec Platon ", écrit Jean Grondin dans son Introduction à la métaphysique, P.U.M., Montréal, 2004, p. 310. 
mathématiques peuvent jouer pour la métaphysique «le rôle de modèles où observer comment les choses viennent à l'existence ${ }^{5}$ ».

\section{La voie «mathématique»}

Cette seconde approche envisage une réflexion mathématique un peu plus poussée sur la paire essence-existence. Elle mettrait ainsi à profit la véritable identité de nature qui existe entre les genèses "verticales" (dialectique $\rightarrow$ mathématiques) de l'essai de 1939 et les genèses " horizontales » (essence $\rightarrow$ existence) de la thèse principale. Lautman est on ne peut plus clair à cet égard: "[...] aucune différence de nature ne sépare la genèse des théories mathématiques à partir de la Dialectique, des genèses qui s'opèrent, au sein des mathématiques, des structures aux existences ${ }^{6}$ ». Les genèses horizontales sont purement mathématiques, et Lautman en donne plusieurs exemples avec lesquels on pourrait immédiatement travailler. Parmi les théories considérées, le couple surface-intégrales fournirait certainement le cas le plus riche et le plus intéressant, s'il n'impliquait une technicité considérable. À cet égard, la paire groupe-représentations me semble plus pure et donc plus accessible. Du reste, dans tous les exemples cités par Lautman, on retrouve toujours la même idée: l' «essence» lautmanienne correspond en gros à ce que les scolastiques appelaient une "propriété nécessaire ${ }^{7}$ ". Dans le cas des groupes, l'essence (au sens traditionnel ou scolastique) est constituée des axiomes de groupe, tandis que la propriété essentielle (c'est-à-dire l'essence ou la structure selon Lautman), c'est la division en classes de conjugaison. C'est cette division, découlant de l'essence du groupe, qui contrôlerait l'existence des caractères, ainsi que leur nombre. Cette «division créatrice", à l'origine d'une genèse horizontale, peut-elle nous donner quelque idée de cette autre genèse qu'est le passage de la dialectique aux mathématiques?

\section{La voie «problématique»}

Si la première voie misait sur la philosophie et la seconde sur les mathématiques, la troisième est résolument "philosophico-mathématique ». S’y engager impliquera une réflexion à partir de certaines "anomalies » qu'une lecture de Lautman permettra de localiser. J'en donne pour principal exemple le passage suivant, qui est rarement cité par les commentateurs. C'est à la fin

5. Lautman 2006, p. 242.

6. Je souligne. Relire à ce sujet la conclusion de l'essai de 1939 dans Lautman, 2006, p. 256 et 257.

7. Voir Breton, Stanislas, Approches phénoménologiques de l'idée d'être, Emmanuel Vite, Paris 1959, p. 41. Évidemment, Lautman n'est pas «essentialiste», et il partage, il me semble, l'antiaristotélisme de son maitre Brunschvicg. 
d'une discussion serrée d'un certain problème dialectique que Lautman écrit :

L'idée de comparer les rapidités de croissance à l'infini de deux quantités constamment croissantes, et liées l'une à l'autre, est rationnellement antérieure à la distinction du continu et du discontinu. Elle peut donc se réaliser en des théories aussi distinctes que la théorie de la croissance des fonctions entières et la théorie de la répartition des nombres premiers ${ }^{8}$.

Cette "antériorité rationnelle", qui admet plus d'une interprétation, n'a rien de limpide, et elle présente il me semble un écart sensible par rapport aux deux thèses, écart qui n'a pas été suffisamment reconnu et expliqué. Comment affirmer que l'idée somme toute assez technique de comportement asymptotique est antérieure à la distinction entre le continu et le discret - distinction "ancienne" s'il en est une - alors que les notions qu'elle met en jeu semblent appartenir aux mathématiques? Et comment au juste doit-on l'articuler en un couple de notions opposées? Et d'ailleurs, les Idées dialectiques impliquent-elles nécessairement des notions en opposition? Lautman n'écrit-il pas dans son travail posthume que la « dialectique semble constituée principalement par des couples de contraires ${ }^{9} »$ ?

La question sous-jacente se rattache à celle de la nature des "problèmes logiques» et de la hiérarchie qui existerait au sein de l'ensemble de ces problèmes. Loin d'aller jusqu'à proposer la réalisation entière du "programme de Lautman » - programme qui préconise justement l'établissement d'une nouvelle théorie des idées - je suggère au contraire de réfléchir à la faisabilité a priori d'un tel programme: "[I]l n'est pas exclu qu'il soit possible, et c'est la seconde des tâches assignables à la philosophie mathématique, d'établir une hiérarchie des idées et une théorie de la genèse des idées les unes à partir des autres, comme l'avait envisagé Platon ${ }^{10}$.» Rappelons que les problèmes dialectiques ne sont rigoureusement connus, au-delà de la simple conscience de leur urgence, que par le truchement des théories mathématiques. Mais le philosophe, s'il recherche l'exactitude, et s'il tente de constituer une dialectique, une théorie de la composition et de la dissociation des notions, échouera en tant que philosophe, puisqu'il se métamorphosera nécessairement en mathématicien. Si le programme de Lautman est réalisable, ce ne sera certes pas en extériorité par rapport aux mathématiques, car il n'y a pas de dialectique pure. La découverte d'une hiérarchie et de relations entre les idées ne pourra être validée qu'au sein des mathématiques. Hors mathématiques, une telle dialectique ne pourra jamais être conçue que comme problématique pure. Et cela devrait faire douter, à mon

8. Lautman, 2006, p. 255. Je souligne.

9. Ibid., p. 276.

10. Lautman, dans Cavaillès, Jean, Euvres complètes de philosophie des sciences, Paris, Hermann, 1994, p. 609. 
sens, de la possibilité de réaliser un pareil programme ${ }^{11}$. De là, peut-être, la grande prudence de Lautman: «il n'est pas exclu» qu'une pareille théorie des idées soit réalisable...

\section{Conclusion}

Récapitulons. Je propose d'étudier la connexion dialectique-mathématique par le biais de deux rapports dont Lautman nous dit qu'ils en sont des analogues: le rapport Dasein-Monde dans la philosophie de Heidegger, et le schéma de genèse essence-existence, dans ses multiples incarnations mathématiques. De plus, je note l'urgence de clarifier la distinction entre notion dialectique et notion mathématique, et conséquemment de revenir sur le concept d'antériorité, que cette dernière soit de nature ontologique, transcendantale ou autre.

Il faut espérer que ces trois enquêtes, bien distinctes dans leur style, fourniront un noyau dur de résultats propres à faire progresser les études lautmaniennes, et plus généralement la philosophie mathématique.

Il y a un dernier point sur lequel il convient d'attirer l'attention. La qualité pédagogique de ces enquêtes devra être proportionnée à la profondeur de l'œuvre étudiée. Le mathématicien Maurice Fréchet réclamait à bon droit de notre philosophe des explications «en langage ordinaire ${ }^{12}$ ». Mathématiciens et philosophes devront avoir les uns pour les autres le souci de la lisibilité et retrouver le bonheur d'expression et le sens pédagogique qui caractérisent les meilleures pages d'Albert Lautman.

11. Comment déterminer par exemple si l'antériorité rationnelle rapportée par Lautman dans la citation de la page 255 est vérifiée dans tout contexte mathématique où elle a un sens? Si la hiérarchie des problèmes est validée par plongement au sein des théories mathématiques, elle est incertaine, car l'histoire des mathématiques est imprévisible; si par contre elle est purement dialectique, son existence contredit l'opinion exprimée par Lautman dans de nombreux passages de son œuvre, opinion très clairement réaffirmée devant le philosophe Jean Hyppolite en 1939, en réponse aux interrogations de ce dernier quant à la nature de la «dialectique » de Lautman.

12. Lautman, 2006, p. 260. 